\title{
Revestimento biodegradável à base de Spirulina platensis na conservação pós-colheita de goiaba Paluma mantidas sob diferentes temperaturas de armazenamento
}

\section{Biodegradable recovery based on Spirulina platensis on post-harvest conservation of goiaba Paluma under different storage temperatures}

\author{
Elny A. Onias*, Albert E. M. M. Teodosio, Marinês P. Bomfim, Railene H. C. Rocha, \\ José F. de Lima e Maria L. S. de Medeiros
}

\author{
Universidade Federal de Campina Grande, Centro de Ciências e Tecnologia Agroalimentar, campus Pombal, PB, Brasil \\ (*E-mail:elnyonias@hotmail.com) \\ http://dx.doi.org/10.19084/RCA17201
}

Recebido/received: 2017.08 .08

Recebido em versão revista/received in revised form: 2018.04.11

Aceite/accepted: 2018.04.12

\section{R E S U M O}

Spirulina platensis é uma microalga rica em proteínas que apresenta composição apropriada para utilização como complemento alimentar.. O objetivo deste trabalho foi avaliar diferentes concentrações de Spirulina platensis como revestimento biodegradável na conservação pós-colheita de goiaba 'Paluma' sob armazenamento refrigerado e ambiente. O ensaio foi desenvolvido na Universidade Federal de Campina Grande, Centro de Ciências e Tecnologia Agroalimentar e instalado em delineamento inteiramente casualizado, em esquema fatorial $2 \times 4 \times 5$, utilizando dois tipos armazenamento (a $10 \pm 2^{\circ} \mathrm{C}$, com $85 \pm 5 \%$ UR e a $24 \pm 2^{\circ} \mathrm{C}$, com $85 \pm 5 \%$ UR), quatro concentrações da microalga $(0 \%, 1 \%$, $2 \%$ e 3\%) e cinco períodos de avaliação (0, 3, 6, 9 e 12 dias). Os resultados mostraram que os revestimentos com Spirulina platensis foram eficientes no retardo da perda de massa nos frutos em ambas as temperaturas de armazenamento, sendo o tratamento $\mathrm{T} 2$, que se refere a concentração de $1 \%$ a $10 \pm 2^{\circ} \mathrm{C}$ o mais eficiente, proporcionando as menores perdas quando comparado a testemunha. Quanto às demais características de qualidade do fruto não foram observadas influências significativas dos revestimentos.

Palavras-chave: Psidium guajava L, microalga, vida útil, maturação, conservação.

\begin{abstract}
A B S T R A C T
Spirulina platensis is a microalgae rich in proteins that has a composition suitable for use as a food supplement. The objective of this study was to evaluate different concentrations of Spirulina platensis as a biodegradable coating on postharvest conservation of 'Paluma' guava under refrigerated storage and environment. The experiment was carried out at the Federal University of Campina Grande, Agro-Food Science and Technology Center and installed in a completely randomized design, in a $2 \times 4 \times 5$ factorial scheme, using two types of storage (at $10 \pm 2{ }^{\circ} \mathrm{C}, 85 \pm 5 \%(0,1,2$ and $3 \%)$ and five evaluation periods (0, 3, 6, 9 and 12 days) ). The results showed that the coatings with Spirulina platensis were efficient in delaying the loss of mass in fruits at both storage temperatures, with the T2 treatment, which refers to a concentration of $1 \%$ at $10 \pm 2{ }^{\circ} \mathrm{C}$, more efficient, providing the lowest losses when compared to the control. Regarding the other quality characteristics of the fruit, no significant influences of the coatings were observed.
\end{abstract}

Keywords: Psidium guajava L., microalgae, shelf life, maturation, conservation. 


\section{INTRODUÇÃO}

A goiabeira (Psidium guajava L.) da família Myrtaceae originária da América Central e do Sul, é uma cultura muito resistente, que tolera altas temperaturas e seca, principalmente na região do semiárido (Forato et al., 2015), apresenta uma produção satisfatória durante todo o ano, sendo bastante popular devido à sua disponibilidade. Produz frutos doces e saborosos, com elevados valores nutricionais e medicinais, preço acessível e de fácil comercialização, tendo uma boa aceitação dos consumidores (Nimisha et al., 2013).

Apresentam o segundo maior teor de ácido ascórbico (vitamina $\mathrm{C}$ ) de todas as frutas pertencentes a sua família. Devido ao seu valor nutricional, podem ser obtidos vários produtos através do seu processamento, tais como: fatias enlatadas, concentrados, desidratados, geleias, sucos, néctares, purés e xaropes (Patil et al., 2014). Entretanto a goiaba também é amplamente consumida in natura, contudo como apresenta uma alta taxa respiratória amadurece rapidamente, entrando em senescência durante o armazenamento à temperatura ambiente (Hong et al., 2012; Vishwasrao \& Ananthanarayan, 2016).

Por se tratar de uma fruta frágil, a comercialização da goiaba ainda está restrita aos mercados locais, em sua produção ainda são utilizadas técnicas de manuseio e transporte inadequadas, bem como a aplicação de práticas de manipulação pós-colheita deficientes, o que acarreta várias alterações na composição da fruta, nomeadamente, danos físicos e podridões.

Atualmente um dos grandes desafios da fruticultura brasileira é a preservação da qualidade da fruta após a colheita. A crescente procura para exportação e mercado interno, fato que o aumento da produção não tem sido suficiente, implica na necessidade de investimento em tecnologias na cadeia produtiva visando minimizar as perdas pós-colheita (Rana et al., 2015; Botelho et al., 2016).

Diante desse cenário, há a necessidade da adoção de técnicas que mantenham e prolonguem a vida pós-colheita dos frutos. Existem diferentes técnicas para a manutenção da qualidade de frutas e hortaliças, dentre as quais destaca-se a aplicação de revestimentos poliméricos, refrigeração, atmosfera modificada e controlada, irradiação (Almeida, 2010; Li et al., 2018).

A utilização da Spirulina platensis vem sendo estudada devido aos seus múltiplos benefícios para a saúde humana (Michalak et al., 2017). Trata-se de uma microalga, cuja composição permite ser utilizada como complemento alimentar, destacando-se na sua composição, alta concentração de proteína entre 60 e 70\%, aminoácidos essenciais, altas concentrações de vitaminas A, B12 e $\beta$-caroteno, bem como 4 a $7 \%$ de lipídios, sendo esses ácidos graxos essenciais, exemplo, ácido linoleico, ácido linolênico, ômega 3 e 6, e ácidos graxos poli-insaturados (Figueira, 2010; Koru, 2012; Pentón-Rol et al., 2018).

A Spirulina possui várias aplicações na área agronômica. Por esse motivo vem sendo intensamente estudada em novas linhas de pesquisas, como o desenvolvimento de biofilmes estruturais, para o recobrimento de frutos na fase de pós-colheita. Muitos estudos têm focado na biofertilização e bioestimulação de plantas na fase de cultivo (Godlewska et al., 2016; Michalak et al., 2017; Salehi et al., 2017).

Recentemente, vem despertando o interesse para o desenvolvimento de componentes que aumente a vida útil dos frutos, por entender que os frutos são extensões dos vegetais, e respondem as aplicações de biomassas em suas superfícies (Onias et al., 2016; Queiroga, 2016). Torna-se importante conhecer os mecanismos da interação do biofilme de recobrimento, com a casca do fruto, e os benefícios conferidos a parte interna, aumento do nível de compostos e conservação da qualidade total (Xing et al., 2016; Oliveira et al., 2018).

As propriedades da biomassa de Spirulina vão muito além da nutrição, seja humana ou vegetal, apresenta em seu conteúdo algo em torno de 15\% das ficobiliproteínas, complexo proteico formado por (ficocianina, ficoeritrina e aloficocianina) (Pentón-Rol et al., 2018) que é responsável pelo aumento de imunidade em animais e plantas. Possui ainda inúmeros componentes peptídicos pouco ainda estudados, que são responsáveis pela sinalização dos processos celulares (Lage-Yusty et al., 2013; Cuellar-Bermudez et al., 2015; Lisboa \& 
Costa, 2016; Yu et al., 2016; Saboya et al., 2017) Por fim, a Spirulina é um organismo que tem muitas aplicabilidades no que se refere a tecnologia agroalimentar.

Neste contexto, objetivou-se com este trabalho avaliar diferentes concentrações de Spirulina platensis como revestimento biodegradável na conservação pós-colheita de goiaba 'Paluma' sob armazenamento refrigerado e ambiente.

\section{MATERIAL E MÉTODOS}

O ensaio foi desenvolvido na Universidade Federal de Campina Grande (UFCG), Centro de Ciências e Tecnologia Agroalimentar (CCTA). Instalado no laboratório de Pós-Colheita de Frutos e Hortaliças, e as análises efetuadas no Laboratório de Análise e Bioquímica de Alimentos. Os frutos de goiabas utilizados foram adquiridos num pomar comercial localizado nas Várzeas de Sousa - PB, distante $47 \mathrm{~km}$ do município de Pombal-PB.

A colheita foi realizada no período da manhã, onde os frutos foram pré-selecionados no campo, excluindo-se os que apresentavam sinais de doenças, presença de patógenos ou algum dano mecânico. Em seguida procedeu-se a higienização dos frutos com uma solução de detergente a $1 \%$ e solução de hipoclorito de sódio 200 ppm de cloro ativo, com imersão de 20 minutos, e posterior enxague em água, depois secados ao ar livre.

A instalação do ensaio foi efetuada em delineamento inteiramente casualizado, em esquema fatorial $2 \times 4 \times 5$, dois tipos de armazenamento, sendo respectivamente refrigerado a $10 \pm 2^{\circ} \mathrm{C}$ com $85 \pm 5 \%$ HR e temperatura ambiente a $24 \pm 2{ }^{\circ} \mathrm{C}$ com $85 \pm 5 \%$ HR. E testados em quatro concentrações das soluções preparadas a partir da biomassa de Spirulina platensis $(0 \%, 1 \%, 2 \%$ e $3 \%)$ e água destilada. As avaliações foram realizadas em cinco períodos $(0,3,6,9$ e 12 dias), com três repetições e cada repetição constituída de dois frutos.

Os tratamentos foram constituídos de acordo com as diferentes concentrações da solução a base da biomassa de Spirulina platensis, compreendidos $\mathrm{T} 1$ - testemunha $(0 \%), \mathrm{T} 2-1 \%, \mathrm{~T} 3-2 \%$ e $\mathrm{T} 4-3 \%$ da solução. Em seguida, os frutos foram imersos durante 20 minutos, sob agitação constante, seguido de secagem à temperatura ambiente, e acondicionados em bandejas de polipropileno e armazenados às temperaturas de $10 \pm 2^{\circ} \mathrm{C}$ e de $24 \pm$ $2^{\circ} \mathrm{C}$, respectivamente. Em cada tempo de avaliação, os frutos foram centrifugados e avaliados quanto aos seguintes parâmetros:

Perda de Massa: determinada para cada fruto em balança semi-analítica com precisão de $\pm 0,01 \mathrm{~g}$. Os resultados foram expressos em perdas percentuais, utilizando-se a relação entre a massa em cada dia de avaliação e a massa inicial, seguindo a equação: \%PM = ((MI - MF) / MI $)$ X100, em que:

$\% \mathrm{PM}=$ percentagem de perda de massa parcial acumulada

$\mathrm{MI}=$ Massa inicial da amostra no período determinado $(\mathrm{g})$

MF = Massa final da amostra no período seguinte a MI (g)

Cor da epiderme - medida através de colorímetro digital, utilizando o Sistema CIELAB, que define um espaço cromático tridimensional com 3 eixos, em coordenadas retangulares $\left(L^{*} a^{*} b^{*}\right)$ que indicam, respectivamente, a luminosidade $\left(\mathrm{L}^{*}\right)$, os tons de vermelho ( $a^{*}$ positivo) a verde (-a* negativo), e os tons amarelo ( $b^{*}$ positivo) a azul ( $-b^{*}$ negativo) e define também em coordenadas cilíndricas $\left(L^{*}, C^{*}, h^{*}\right)$.

Os valores de "a" $\mathrm{e}$ " $\mathrm{b}$ " foram convertidos em ângulo Hue " $h$ " ( $\left.h=\tan ^{-1}(b / a)\right)$, que representa a intensidade da cor, e croma $\left(\mathrm{C}=\left(\mathrm{a}^{2}+\mathrm{b}^{2}\right)^{1 / 2}\right)$ a pureza da cor.

Firmeza dos frutos - avaliada em texturómetro (FRUIT HARDNESS TESTER), com profundidade de penetração de $2,0 \mathrm{~mm}$, velocidade de $2,0 \mathrm{~mm} \mathrm{~s}^{-1}$ e ponteiro, TA 8/1000. As leituras foram realizadas nos frutos inteiros, tomando-se quatro medidas por fruto, em faces opostas, após a remoção de porções da casca. Os resultados obtidos foram expressos em Newtons (N).

$\mathrm{pH}$ - determinado através de $\mathrm{pHmetro}$ digital de bancada por leitura direta na polpa homogeneizada e diluída em água, conforme IAL (2008). 
Acidez Titulável (AT) - determinada de acordo com metodologia recomendada pelo Instituto Adolfo Lutz (IAL, 2008), utilizando-se 10 gramas de polpa homogeneizada e diluída em $100 \mathrm{~mL}$ de água destilada, seguida de titulação com solução padronizada de $\mathrm{NaOH}$ 0,1N, usando como indicador o ponto de viragem da fenolftaleína. Os resultados foram expressos em $\mathrm{g}$ de ácido cítrico $100 \mathrm{~g}^{-1}$ da amostra.

Sólidos Solúveis (SS) - avaliado diretamente da polpa homogeneizada, por leitura em refratómetro digital (modelo PR - 100, Palette, Atago Co., LTD., Japan), expressos em ${ }^{\circ}$ Brix (AOAC, 2005).

Vitamina C - determinada por espetrofotometria, pelo método de Terada et al. (1978). Para determinação da vitamina $C$ foi utilizado $300 \mathrm{mg}$ da amostra triturada e mix. Para as leituras foi utilizado espectrofotômetro digital utilizando o comprimento de onda de $525 \mathrm{~nm}$. Os resultados foram comparados com a curva padrão de ácido ascórbico sendo expresso em $100 / \mathrm{\mu g} / \mathrm{mL}^{-1} \mathrm{em}$ ácido oxálico $0,5 \%$, construída da mesma maneira das amostras.

\section{RESULTADOS E DISCUSSÃO}

A composição da biomassa da Spirulina platensis possui muitos compostos que promovem plastificação porosa e grade de interação com complexos proteicos, a exemplo PHBV (Polihidroxi butirato Valerato) permite a formação de cadeias de estruturação mecânica e interativa na superfície do fruto (Moraes et al., 2015; Torres et al., 2015; Teuling et al., 2017). As concentrações de proteínas, carboidratos, aminoácidos e minerais são apresentados nos Quadros 1 e 2. Parte dessas substâncias são responsáveis por promover efeitos bioativos em frutos, principalmente na regulação da transpiração e redução de perda de massa (Oliveira et al., 2018), as soluções empregadas no recobrimento dos frutos de Goiaba apresentaram uma boa solubilidade em água, indicando que o biofilme formado apresentou um bom desempenho em relação a interação de complexos gelatinosos; e cadeias estruturais no recobrimento. As soluções preparadas a partir da biomassa de Spirulina foram de fácil formulação, por conter maior quantidade de proteínas com alta solubilidade em água, e outra parte hidrofóbica formando uma grade micelar para a estruturação do biofilme (Quadro 1). Os demais constituintes da biomassa de Spirulina atuaram como adjuvantes da estrutura cristalina, que refletiram na boa aparência do fruto após aplicação.

Quadro 1 - Composição da biomassa de Spirulina plantensis

\begin{tabular}{cc}
\hline Determinação & Resultado \\
\hline Umidade e voláteis $(\mathrm{g} / 100 \mathrm{~g})$ & $6,81(0,05)^{\mathrm{a}}$ \\
Cinzas $(\mathrm{g} / 100 \mathrm{~g})$ & $24,15(0,28)^{\mathrm{a}}$ \\
Lipídeos $(\mathrm{g} / 100 \mathrm{~g})$ & $11,48(0,20)^{\mathrm{a}}$ \\
Nitrogênio $(\mathrm{g} / 100 \mathrm{~g})$ & $4,61(0,01)^{\mathrm{a}}$ \\
Proteínas $(\mathrm{g} / 100 \mathrm{~g})$ & $28,82(0,05)^{\mathrm{a}}$ \\
Carboidratos totais $9 \mathrm{Kcal} / 100 \mathrm{~g})$ & $334 \mathrm{c}$ \\
pH (solução $10 \%)$ & $8,15(0,02)^{\mathrm{a}}$ \\
Cálcio $(\mathrm{mg} / \mathrm{Kg})$ & $55689(0,05)^{\mathrm{a}}$ \\
Cobalto $(\mathrm{mg} / \mathrm{Kg})$ & $0,49(0,02)^{\mathrm{a}}$ \\
Cobre $(\mathrm{mg} / \mathrm{Kg})$ & $142(2)^{\mathrm{a}}$ \\
Enxofre $(\mathrm{mg} / \mathrm{Kg})$ & $4298(143)^{\mathrm{a}}$ \\
Ferro $(\mathrm{mg} / \mathrm{Kg})$ & $355(1)^{\mathrm{a}}$ \\
Fósforo $(\mathrm{mg} / \mathrm{Kg})$ & $14541(229)^{\mathrm{a}}$ \\
Magnésio $(\mathrm{mg} / \mathrm{Kg})$ & $22547(638)^{\mathrm{a}}$ \\
Manganês $(\mathrm{mg} / \mathrm{Kg})$ & $131(1)^{\mathrm{a}}$ \\
Níquel $(\mathrm{mg} / \mathrm{Kg})$ & $0,76(0,01)^{\mathrm{a}}$ \\
Potássio $(\mathrm{mg} / \mathrm{Kg})$ & $10042(90)^{\mathrm{a}}$ \\
Sódio $(\mathrm{mg} / \mathrm{Kg})$ & $4879(63)^{\mathrm{a}}$ \\
Zinco $(\mathrm{mg} / \mathrm{Kg})$ & $11,83(0,83)^{\mathrm{a}}$ \\
\hline
\end{tabular}

Quadro 2 - Composição de aminoácidos da biomassa de Spirulina platensis

\begin{tabular}{cc}
\hline Aminoácidos totais & $\mathrm{g} / \mathbf{1 0 0 \mathrm { g }}$ \\
\hline Ácido aspártico & 1,33 \\
Ácido glutâmico & 3,37 \\
Serina & 1,36 \\
Glicina & 1,26 \\
Histidina & 0,60 \\
Arginina & 2,42 \\
Treonina & 1,58 \\
Alanina & 1,44 \\
Prolina & 1,62 \\
Tirosina & 0,97 \\
Valina & 1,43 \\
Metionina & 0,39 \\
Cistina & 0,13 \\
Isoleucina & 1,38 \\
Leucina & 1,83 \\
Fenilalanina & 1,24 \\
Lisina & 1,49 \\
triptofano & 0,16 \\
\hline &
\end{tabular}


De acordo com os dados obtidos, houve interação significativa $(p<0,05)$ entre os revestimentos e tempo de armazenamento para perda de massa, firmeza, sólidos solúveis e acidez titulável dos frutos armazenados na temperatura de $24^{\circ} \mathrm{C}$ (Quadro 3). As variáveis Luminosidade, ângulo hue, cromaticidade e vitamina C apresentaram diferença significativa para o tempo de armazenamento, enquanto para a vitamina $C$ houve efeito significativo do tempo e do recobrimento de forma isolada. Para a outra condição de temperatura $\left(10^{\circ} \mathrm{C}\right)$ foi observada interação significativa para perda de massa, cromaticidade, acidez titulável e vitamina $C$, as demais variáveis apresentaram efeito do tempo de armazenamento de forma isolada.

Observou-se que a perda de massa dos frutos aumentou gradativamente durante o período de armazenamento e que em ambas as condições de armazenamento as maiores perdas foram para os frutos sem aplicação de revestimento (T1). As menores perdas de massa foram obtidas para o tratamento T2 no armazenamento refrigerado (Figura 1a), o qual atingiu valores inferiores a $8 \%$ aos 12 dias de armazenamento, enquanto que para o armazenamento sob temperatura ambiente as menores foram para o tratamento T4 (Figura 1b).
A perda de massa em frutas e vegetais in natura ocorre devido à perda de água causada por processos de transpiração e respiração (Zhu et al., 2008). Forato et al. (2015), avaliaram o efeito de revestimentos comestíveis preparados com goma de cajueiro e carboximetilcelulose na conservação de goiabas frescas e cortadas, também observaram que as amostras não revestidas apresentaram rápida taxa de degradação, enquanto os frutos que receberam revestimentos obtiveram maior tempo de conservação; verificando-se que estes atuaram positivamente, como agente conservante retardando a perda de massa nos frutos.

Observando-se os resultados da luminosidade L* (Figura 2a e 2b) verificou-se um aumento em todos os tratamentos, em ambos os armazenamentos. Os frutos do tratamento T4 mantidos sob refrigeração apresentaram valores inferiores aos demais tratamentos, os mesmos apresentaram coloração mais escura por mais tempo e que, consequentemente, o revestimento atuou na redução do seu metabolismo.

Assim como os frutos do tratamento T4 armazenados sob temperatura ambiente apresentaram o mesmo efeito até sexto dia de armazenamento (Figura 2a e 2b), Queiroga (2016), utilizando

Quadro 3 - Resumo da análise de variância para perda de massa (PM), luminosidade (L), ângulo hue (H), cromaticidade (C), firmeza, pH, sólidos solúveis totais (SST), acidez total titulável (ATT) e vitamina C de de frutos de goiaba revestidos com Spirulina platensis durante o armazenamento a $10 \pm 2{ }^{\circ} \mathrm{C}, 80 \pm 5 \mathrm{HR}$ (a) e $24 \pm 2{ }^{\circ} \mathrm{C}, 80 \pm 5 \mathrm{HR}$

\begin{tabular}{|c|c|c|c|c|c|c|c|c|c|c|c|}
\hline \multicolumn{12}{|c|}{ Ambiente } \\
\hline \multirow{2}{*}{ FV } & \multirow{2}{*}{ GL } & \multicolumn{10}{|c|}{$\mathrm{QM}$} \\
\hline & & PM & L & $\mathrm{H}$ & $\mathrm{C}$ & Firmeza & \multicolumn{2}{|c|}{$\mathrm{pH}$} & SST & ATT & Vitamina C \\
\hline Revestimentos (R) & 3 & $26,975^{* *}$ & $24,807 \mathrm{~ns}$ & 0,336 ns & $19,187 \mathrm{~ns}$ & $4,744 \mathrm{~ns}$ & \multicolumn{2}{|c|}{$0,071 \mathrm{~ns}$} & $0,688 \mathrm{~ns}$ & $0,183^{* *}$ & $53,278^{* *}$ \\
\hline Tempos (T) & 3 & $407,107^{* *}$ & $281,811^{* *}$ & $3,647^{* *}$ & $36,7258^{*} 9$ & $9771,821^{* *}$ & \multicolumn{2}{|c|}{$0,018 \mathrm{~ns}$} & $77,560^{* *}$ & $2,255^{* *}$ & $986,125^{* *}$ \\
\hline $\mathrm{R} \times \mathrm{T}$ & 9 & $3,716^{* *}$ & $12,824 \mathrm{~ns}$ & $0,278 \mathrm{~ns}$ & $6,105 \mathrm{~ns}$ & $9,791^{* *}$ & \multicolumn{2}{|c|}{$0,028 \mathrm{~ns}$} & $1,437^{* *}$ & $0,081^{*}$ & $21,459 \mathrm{~ns}$ \\
\hline Resíduo & 80 & 0,886 & 12,155 & 0,246 & 7,708 & 2,640 & \multicolumn{2}{|c|}{0,027} & 0,297 & 0,034 & 11,002 \\
\hline CV (\%) & - & 18,19 & 5,20 & 0,55 & 6,09 & 7,85 & \multicolumn{2}{|c|}{4,26} & 4,53 & 7,30 & 8,19 \\
\hline \multicolumn{12}{|c|}{ Refrigerada } \\
\hline \multirow{2}{*}{ FV } & \multirow{2}{*}{ GL } & \multicolumn{10}{|c|}{ QM } \\
\hline & & PM & $\mathbf{L}$ & $\mathbf{H}$ & $\mathrm{C}$ & Firm & & $\mathrm{pH}$ & SST & ATT & Vit C \\
\hline Revestimentos (R) & 3 & $117,402^{* *}$ & 15,042 ns & $0,162 \mathrm{~ns}$ & $45,491^{*}$ & 6,70 & & $0,082 \mathrm{~ns}$ & $3,731 \mathrm{~ns}$ & $0,114 \mathrm{~ns}$ & $590,140^{* *}$ \\
\hline Tempos (T) & 3 & $328,324^{* *}$ & $189,065^{* *}$ & $0,420^{* *}$ & $34,777^{* *}$ & 7938, & $95^{* *}$ & $0,615^{* *}$ & $27,239^{* *}$ & $7,853^{* *}$ & $496,680^{* *}$ \\
\hline $\mathrm{R} \times \mathrm{T}$ & 12 & $11,795^{* *}$ & $8,711 \mathrm{~ns}$ & $0,080 \mathrm{~ns}$ & $20,305^{*}$ & 7,44 & & $0,054 \mathrm{~ns}$ & $2,461 \mathrm{~ns}$ & $0,267^{* *}$ & $78,249^{* *}$ \\
\hline Resíduo & 100 & 4,074 & 10,738 & 0,102 & 8,837 & 9,9 & & 0,031 & 1,428 & 0,099 & 27,983 \\
\hline CV (\%) & - & 37,92 & 4,93 & 0,36 & 6,61 & 17 & & 4,68 & 9,34 & 10,65 & 11,88 \\
\hline
\end{tabular}

ns, **, * respectivamente não significativos, significativo a $p<0,01$ e $p<0,05$. 
biofilme à base de Chlorella sp. na qualidade pós-colheita de goiaba 'Paluma' observou que o biofilme na concentração de $2 \%$ aplicado por imersão e armazenado à temperatura ambiente apresentou melhor resultado, indicando que este retardou a maturação dos frutos.

Comportamento semelhante foi observado por Santana (2015), que verificou no início do armazenamento de goiabas 'Paluma', que a luminosidade era da ordem de 55 a 60 e atingiu valores entre 70 e 72 no sexto dia, após a colheita. Resultados similares foram encontrados nessa pesquisa, no início do armazenamento obteve-se valores de $\mathrm{L}^{*} \mathrm{em}$ torno de 62, sendo que estes valores tiveram um aumento, porém não ultrapassando a 72 no final das avaliações. Ainda segundo o mesmo autor, os valores de luminosidade vão-se alterando conforme os frutos vão amadurecendo e sua cor verde-escura passa a uma cor amarelo-claro, o que eleva o brilho e a reflexão da luz sobre a casca, com consequente aumento do parâmetro da luminosidade.

No que se refere à intensidade da cor $\left(h^{*}\right)$, mesmo com pequena oscilação entre valores, houve diferença nos frutos do tratamento $\mathrm{T} 4$, armazenados sob refrigeração e nos frutos do tratamento T3, armazenados sob temperatura ambiente, onde a cor dos
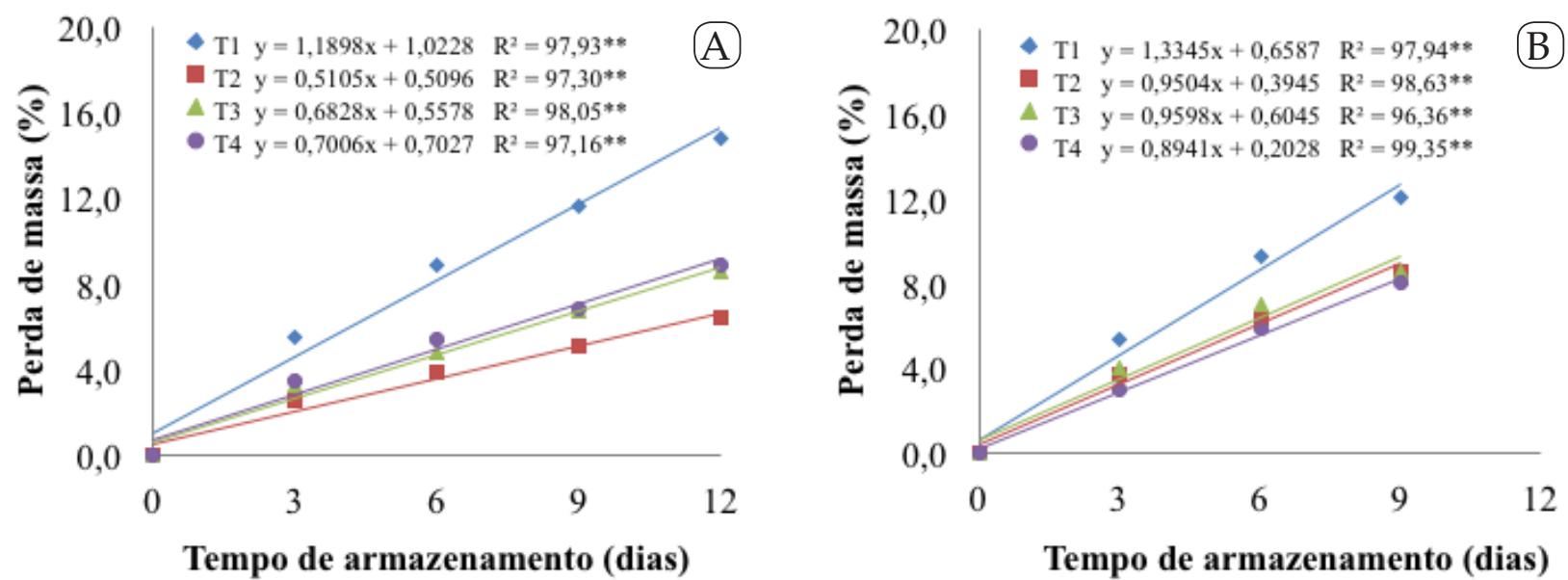

Figura 1 - Perda de massa de frutos de goiaba revestidos com Spirulina platensis durante o armazenamento a $10 \pm 2$ oC, $80 \pm 5$ $\mathrm{HR}\left(\right.$ a) e $24 \pm 2^{\circ} \mathrm{C}, 80 \pm 5 \mathrm{HR}(\mathrm{b})$.
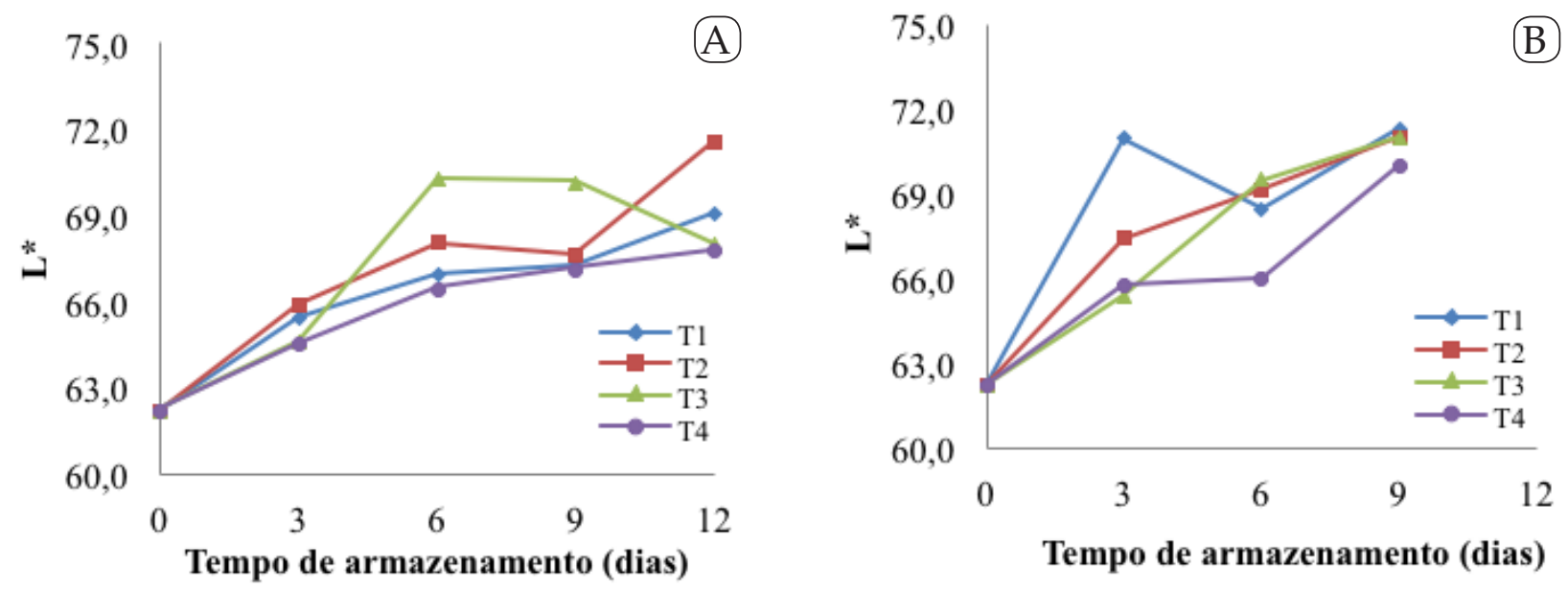

Figura 2 - Luminosidade $L^{*}$ de frutos de goiaba revestidos com Spirulina platensis durante 0 armazenamento a $10 \pm 2$ oC , $80 \pm$ $5 \mathrm{HR}(\mathrm{a})$ e $24 \pm 2^{\circ} \mathrm{C}, 80 \pm 5 \mathrm{HR}(\mathrm{b})$. 
frutos evoluiu mais lentamente do amarelo para o vermelho, indicando que os revestimentos foram mais eficientes na conservação dos frutos (Figura 3a e 3b). Pinheiro (2012), ao avaliar revestimentos de cera de carnaúba em caju e goiaba, verificou que as tonalidades das goiabas armazenadas à temperatura de refrigeração permaneceram praticamente inalteradas até o $16^{\circ}$ dia de armazenamento.

Em relação à pureza da cor Croma $\left(\mathrm{C}^{*}\right)$ ocorreu um pequeno aumento de 0 ao $3^{\circ}$ dia de armazenamento, devido às mudanças na coloração dos frutos nesse período, exceto para os frutos do tratamento T4 para ambas as temperaturas de armazenamento, mostrando a eficiência do mesmo. Os frutos do controle apresentaram valores mais elevados indicando um amadurecimento precoce, em ambos os armazenamentos (Figura 4a e 4b). Comportamento semelhante foi observado por Trindade et al. (2004). De modo geral o croma variou em torno de 42 a 46,5 durante o armazenamento. Esses valores foram semelhantes aos encontrados por Pereira (2009) para a intensidade de cor de goiaba Paluma, que obteve valor de 43,8 na faixa de cor variando do verde amarelado para amarelo.

A firmeza da polpa dos frutos diminuiu com o tempo de avaliação independentemente do
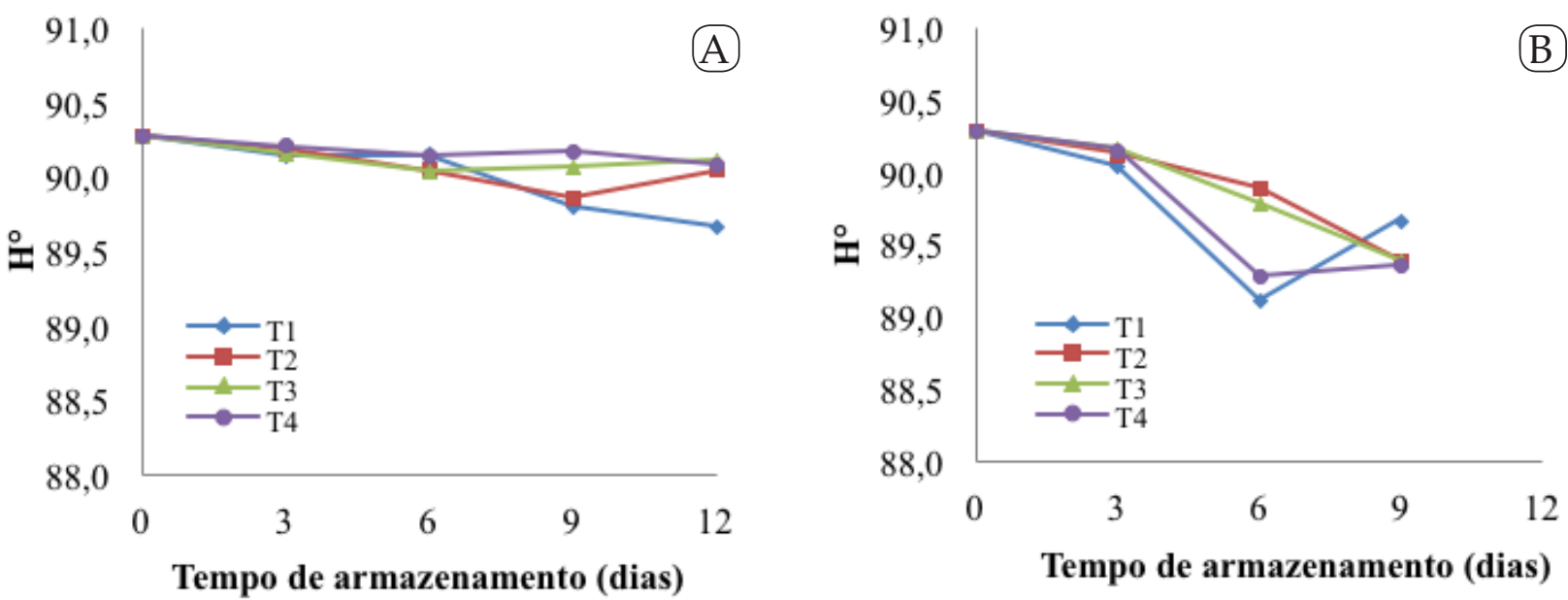

Figura 3 - Ângulo Hue $\mathrm{h}$ * de frutos de goiaba revestidos com Spirulina platensis durante 0 armazenamento a $10 \pm 2$ oC, $80 \pm 5$ $\mathrm{HR}(\mathrm{a})$ e $24 \pm 2^{\circ} \mathrm{C}, 80 \pm 5 \mathrm{HR}(\mathrm{b})$.
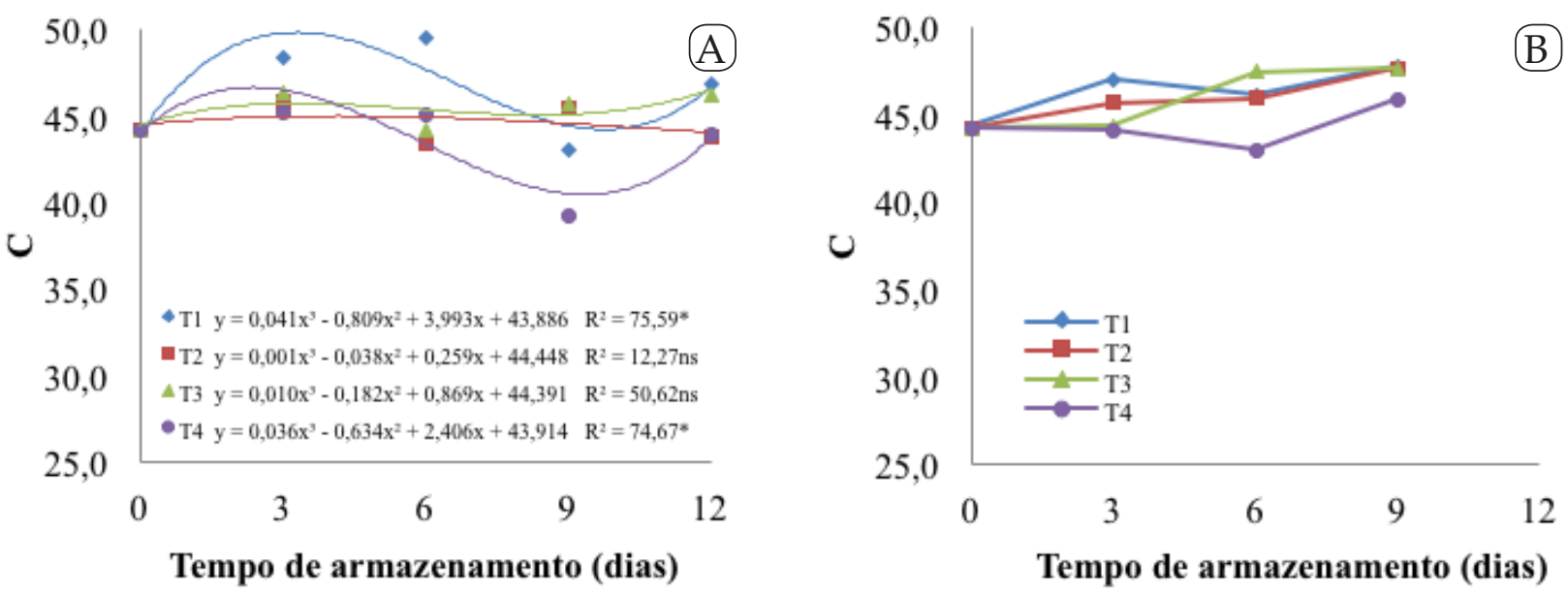

Figura 4 - Croma C* de frutos de goiaba revestidos com Spirulina platensis durante o armazenamento a $10 \pm 2$ o C , 80 5 HR (a) e $24 \pm 2{ }^{\circ} \mathrm{C}, 80 \pm 5 \mathrm{HR}(\mathrm{b})$. 
revestimento dos frutos e da temperatura de armazenamento. Ocorreu uma redução acentuada de 0 ao $3^{\circ}$ dia de armazenamento, em seguida teve uma retenção na perda da firmeza até o último dia de avaliação em ambas as temperaturas (Figura 5a e 5b). A firmeza dos frutos é um dos principais atributos de qualidade julgados pelo consumidor e, portanto, é extremamente importante na aceitação geral do produto. A goiaba sofre uma rápida perda de firmeza durante o seu amadurecimento, o que contribui muito para a sua curta vida útil de prateleira, ficando susceptivel à contaminação por fungos (Hong et al., 2012). As frutas, como a lichia e a manga, também foram relatadas como sendo mais firmes quando tratadas com revestimento à base de quitosana (Lin et al., 2011; Jongsri et al., 2016).

Houve pouca oscilação na variável pH em função do tempo de armazenamento e independentemente dos revestimentos. Observou-se decréscimo de $\mathrm{pH}$ a partir do $3^{\circ}$ dia, seguido por um leve incremento devido a redução da acidez, que ocorreu após esse período para a maioria dos tratamentos sob condição de armazenamento refrigerado. Os frutos apresentaram um valor médio de $\mathrm{pH}$ de 3,80 a 4,0 em ambas as temperaturas (Figura 5a e 5b). Aquino et al. (2015), observou que os valores
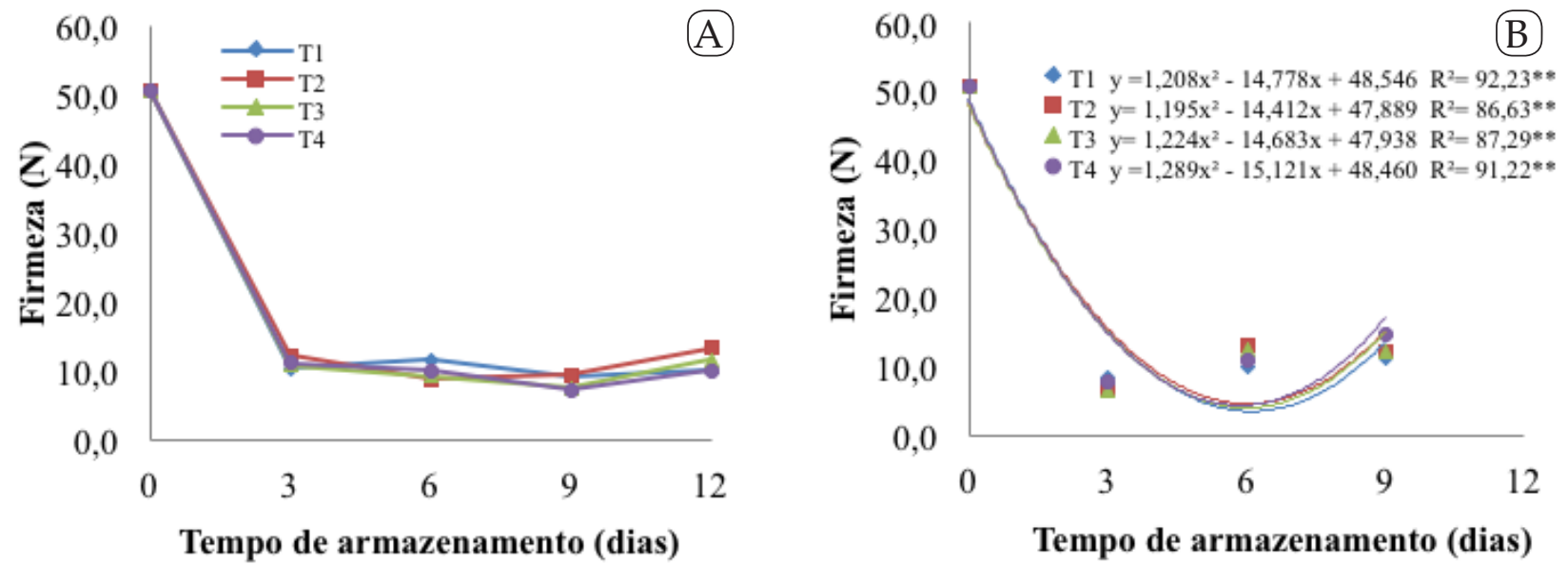

Figura 5 - Firmeza de frutos de goiaba revestidos com Spirulina platensis durante o armazenamento a $10 \pm 2$ oC , 80 5 HR (a) e $24 \pm 2{ }^{\circ} \mathrm{C}, 80 \pm 5 \mathrm{HR}(\mathrm{b})$.
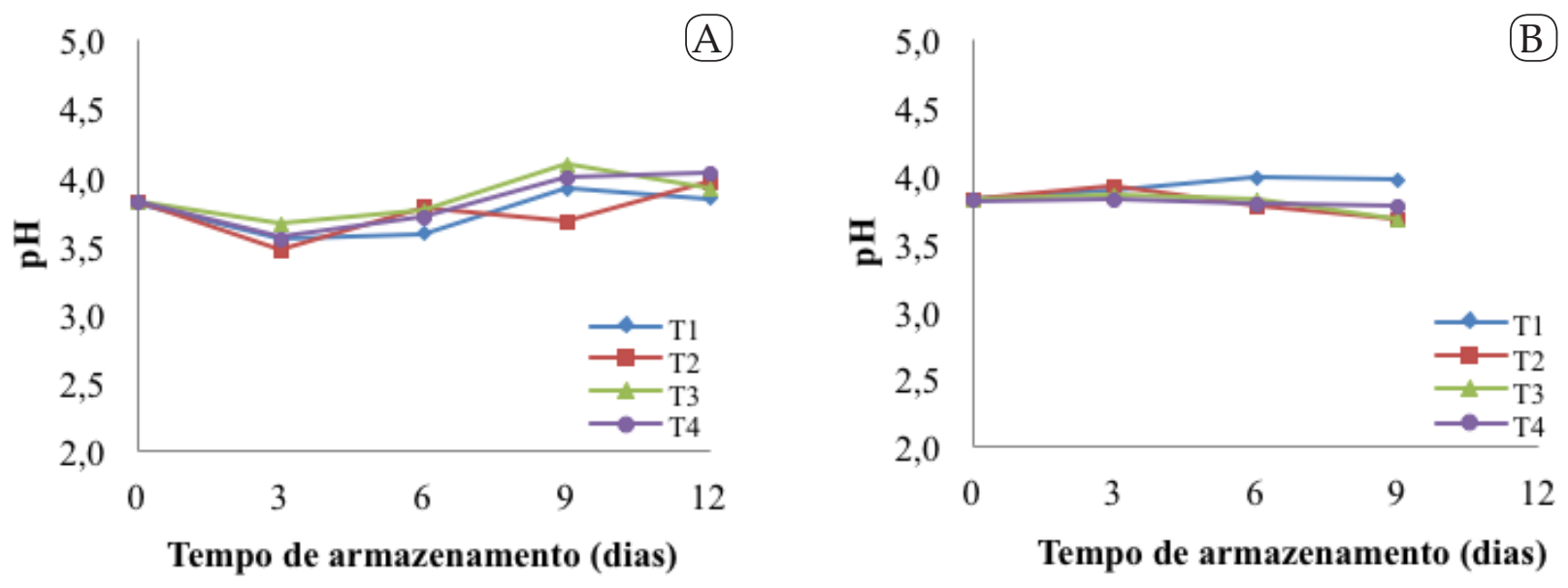

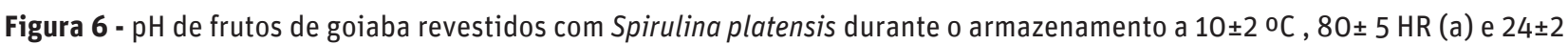
${ }^{\circ} \mathrm{C}, 80 \pm 5 \mathrm{HR}(\mathrm{b})$. 
de $\mathrm{pH}$ em goiabas com revestimento à base de amido de mandioca e quitosana ou sem revestimento, manteve-se entre 3,85 e 4,10, apresentando pequenas diferenças significativas entre os tratamentos ao longo do período de armazenamento.

Os teores de SS comportaram-se de forma quadrática. Observa-se na Figura 7 que houve uma diminuição dos teores durante o armazenamento, e que esta ocorreu de forma mais intensa para os frutos revestidos com comestíveis biofilme(Figura 7a e7b).

O amadurecimento dos frutos é acompanhado por uma série de processos físicos e bioquímicos que resultam na síntese e degradação de pigmentos, conversão de amido em açúcar (Botelho et al., 2016). Segundo Siqueira (2012), a goiaba é um fruto com baixos teores de amido e, por conseguinte, espera-se que não haja grandes alterações no teor de SS ao longo do amadurecimento do fruto. O mesmo autor verificou que a aplicação de revestimentos comestíveis à base de quitosana e alginato de sódio na conservação pós-colheita de goiaba e maracujá-azedo, proporcionaram uma redução nos teores de SS ao longo do tempo de conservação, passando de $12,5^{\circ}$ Brix no início do armazenamento para $9,0^{\circ}$ Brix no final.
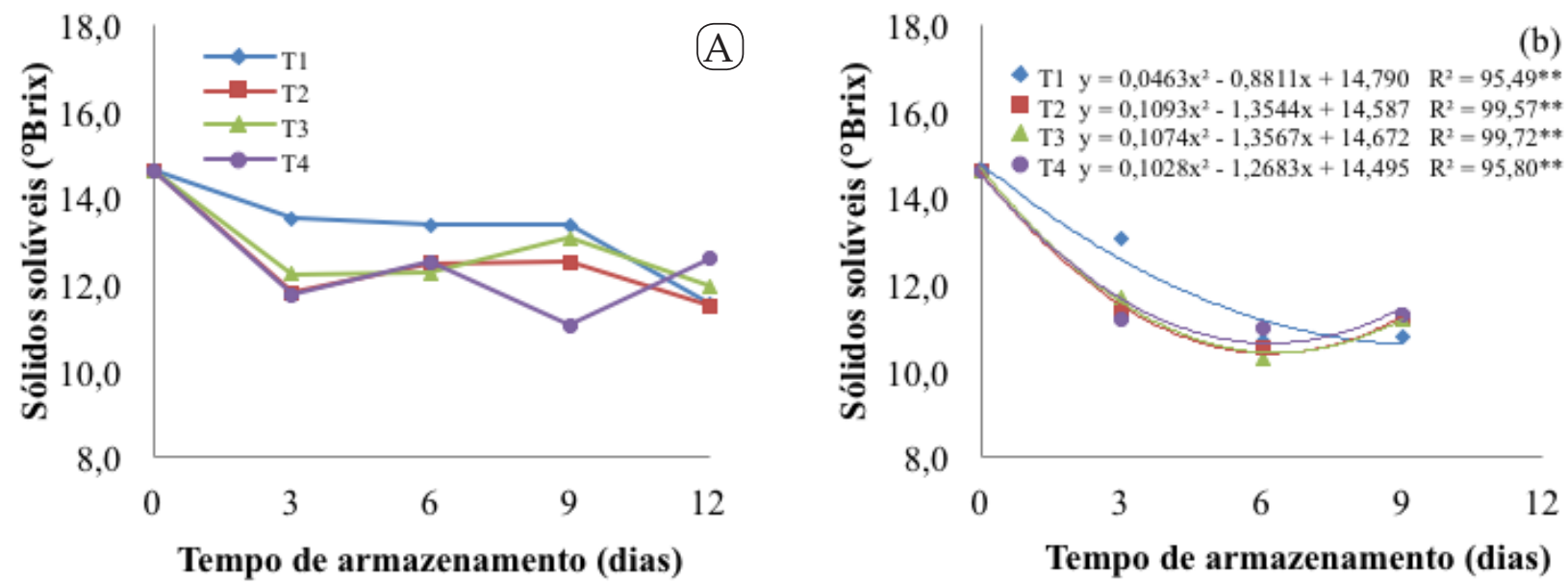

Figura 7 - Teor de sólidos solúveis totais de frutos de goiaba revestidos com Spirulina platensis durante 0 armazenamento a $10 \pm 2{ }^{\circ} \mathrm{C}, 80 \pm 5 \mathrm{HR}(\mathrm{a})$ e $24 \pm 2^{\circ} \mathrm{C}, 80 \pm 5 \mathrm{HR}(\mathrm{b})$.
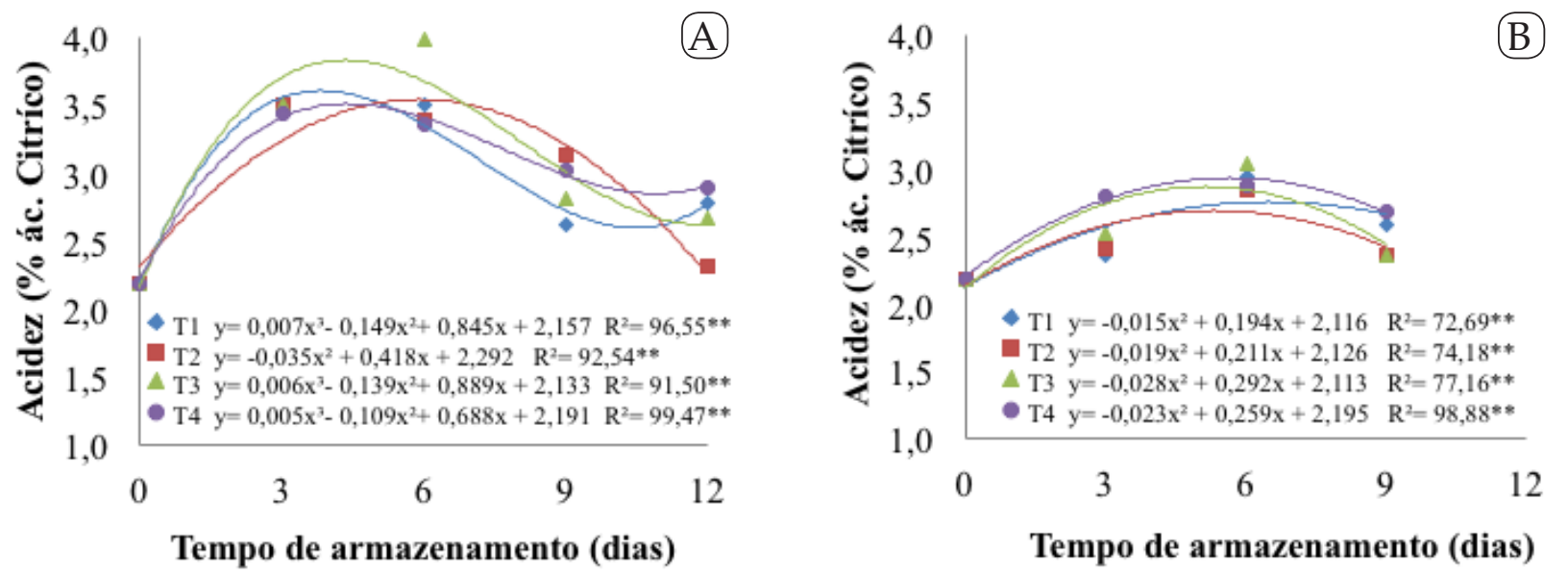

Figura 8 - Acidez titulável total de frutos de goiaba revestidos com Spirulina platensis durante 0 armazenamento a $10 \pm 20 \mathrm{C}$, $80 \pm 5$ HR (a) e $24 \pm 2{ }^{\circ} \mathrm{C}, 80 \pm 5 \mathrm{HR}$ (b). 

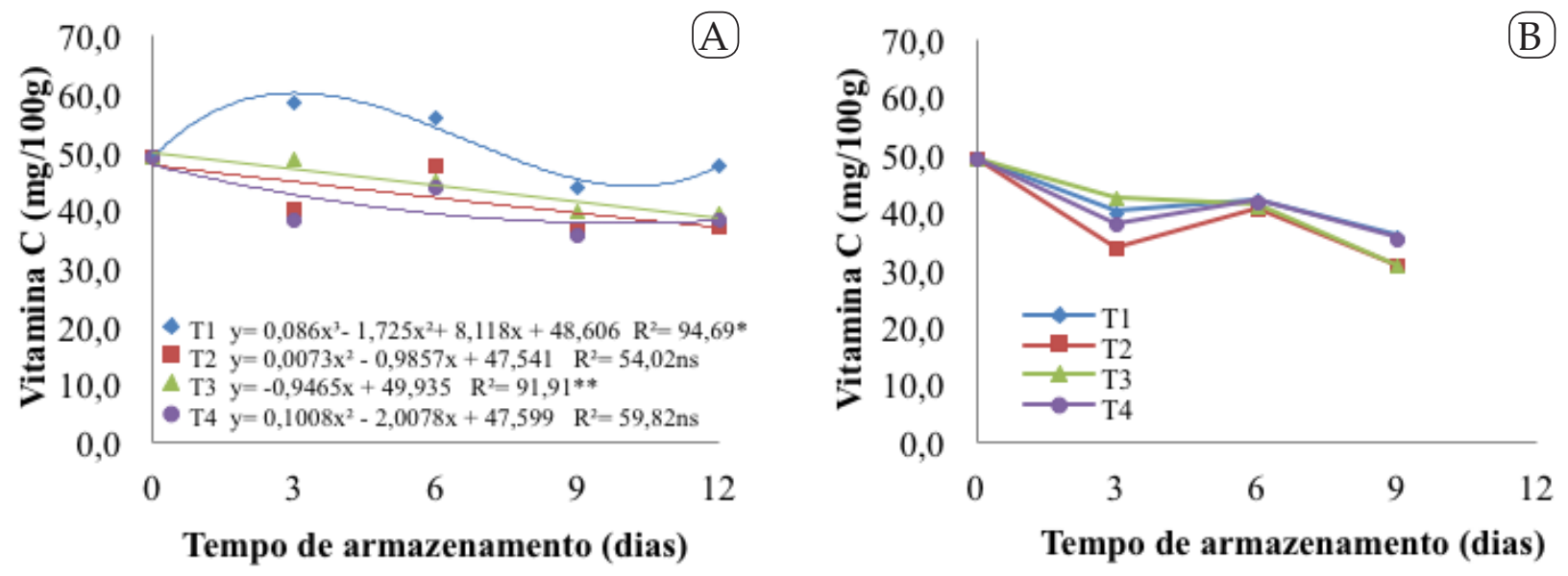

Figura 9 - Vitamina $\mathrm{C}$ de frutos de goiaba revestidos com Spirulina platensis durante o armazenamento a $10 \pm 2 \mathrm{OC}, 80 \pm 5 \mathrm{HR}$ (a) e $24 \pm 2{ }^{\circ} \mathrm{C}, 80 \pm 5 \mathrm{HR}(\mathrm{b})$.

Para a variável AT houve aumento do $3^{\circ}$ para o $6^{\circ}$ dia de armazenamento, com posterior redução em todos os revestimentos em ambas as condições de armazenamento. Esta redução na acidez durante o armazenamento está associada ao processo normal de evolução da maturação, na qual os ácidos orgânicos são metabolizados pela via respiratória e convertidos em moléculas não ácidas. (Figura $8 \mathrm{a}$ e 8b).

Aquino et al. (2015) relataram que as goiabas revestidas com amido de mandioca e quitosana apresentaram os maiores valores de acidez titulável em relação as goiabas não revestidas. Esse comportamento também foi observado por Hong et al. (2012) para goiabas revestidas com quitosana durante o armazenamento a $11^{\circ} \mathrm{C}$ durante 12 dias.

Para a variável vitamina $C$ houve perda gradual ao longo do tempo de avaliações, redução que não foi efetivamente inibida pela utilização dos revestimentos à base de Spirulina platensis, conforme as figuras (Figura 9a e 9b). Hong et al. (2012) observaram que o revestimento a base de quitosana nas concentrações a 1 e $2 \%$ inibiram a redução da vitamina $C$ em goiabas, enquanto que nas concentrações 0,5 e o controle não conseguiram inibir a redução da vitamina $C$.

\section{CONCLUSÃO}

Os revestimentos com Spirulina platensis foram eficientes no retardo da perda de massa nos frutos em ambas as condições de armazenamento, sendo o tratamento $\mathrm{T} 2$, na concentração de $1 \%$ a $10 \pm 2^{\circ} \mathrm{C}$ o mais eficiente, proporcionando as menores perdas de massa quando comparado com o tratamento testemunha. Quanto às demais características de qualidade do fruto não foram observadas influências significativas quando utilizou-se os revestimentos a base de Spirulina.

\section{REFERÊNCIAS BIBLIOGRÁFICAS}

Almeida, D.M. (2010) - Biofilme de blenda de fécula de batata e celulose bacteriana na conservação de fruta minimamente processada. Tese de Doutorado. Curitiba, Universidade Federal do Paraná. 283 p.

AOAC (2005) - Official Methods of Analysis of the Association of Official Analytical Chemists. 18 ed. Gaithersburg, Maryland. Association of Official Analytical Chemists.

Aquino, A.B. de; Blank, A.F. \& Santana, L.C.L.A. de (2015) - Impact of edible chitosan-cassava starch coatings enriched with Lippia gracilis Schauer genotype mixtures on the shelf life of guavas (Psidium guajava L.) during storage at room temperature. Food Chemistry, vol. 171, p. 108-116. https://doi.org/10.1016/j. foodchem.2014.08.077 
Botelho, L.N.S.; Rocha, D.A.; Braga, M.A.; Silva, A. \& Abreu, C.M.P. de (2016) - Quality of guava cv. ‘Pedro Sato' treated with cassava starch and cinnamon essential oil. Scientia Horticulturae, vol. 209, p. 214-220. https://doi.org/10.1016/j.scienta.2016.06.012

Cuellar-Bermudez, S.P.; Aguilar-Hernandez, I.; Cardenas-Chavez, D.L.; Ornelas-Soto, N.; Romero-Ogawa, M.A. \& Parra-Saldivar, R. (2015) - Extraction and purification of high-value metabolites from microalgae: essential lipids, astaxanthin and phycobiliproteins. Microbial Biotechnology. vol. 8, n. 2, p. 190-209. https:// doi.org/10.1111/1751-7915.12167

Figueira, F.S. (2010) - Produção de Pão sem Glúten Enriquecido com Spirulina Platensis. Dissertação (Mestrado em Engenharia e Ciência de Alimentos)-Universidade Federal do Rio Grande, Rio Grande.

Forato, L.A.; Brito, D. de; Rizzo, J. S. de; Gastaldi, T.A. \& Assis, O.B.G. (2015) - Effect of cashew gumcarboxymethylcellulose edible coatings in extending the shelf-life of fresh and cut guavas. Food Packaging and Shelf Life, vol. 5, p. 68-74. https://doi.org/10.1016/j.fpsl.2015.06.001

Godlewska, K.; Michalak, I.; Tuhy, A. \& Chojnacka, K. (2016) - Plant Growth Biostimulants Based on Different Methods of Seaweed Extraction with Water. BioMed Research International, vol. 2016, art. 5973760. http:// dx.doi.org/10.1155/2016/5973760

Hong, K.; Xie, J.; Zhang, L.; Sun, D. \& Gong, D. (2012) - Effects of chitosan coating on postharvest life and quality of guava (Psidium guajava L.) fruit during cold storage. Scientia Horticulturae, vol. 144, p. 172-178. https://doi.org/10.1016/j.scienta.2012.07.002

IAL (2008) - Métodos físico-químicos para análise de alimentos. 4 ed. São Paulo: Instituto Adolfo Lutz.

Jongsri, P.; Wangsomboondee, T.; Rojsitthisak, P. \& Seraypheap, K. (2016) - Effect of molecular weights of chitosan coating on postharvest quality and physicochemical characteristics of mango fruit. LWT - Food Science and Technology, vol. 73, p. 28-36. https://doi.org/10.1016/j.lwt.2016.05.038

Koru, E. (2012) - Earth Food Srpirulina (Arthrospira): Production and Quality Standarts. In: Food Additive. Cap. 11.

Lage-Yusty, M.A.; Caramés-Adán, P. \& López-Hernández, J. (2013) - Determination of phycobiliproteins by constantwavelength synchronous spectrofluorimetry method in red algae. CYTA-Journal of Food, vol. 11, n. 3, p. 243-247. https://doi.org/10.1080/19476337.2012.728629

Li, B.; Lecourt, J. \& Bishop, G. (2018) - Advances in Non-Destructive Early Assessment of Fruit Ripeness towards Defining Optimal Time of Harvest and Yield Prediction-A Review. Plants, vol. 7, n. 3, p. 1-20. https://doi.org/10.3390/plants7010003

Lisboa, C.R. \& Costa, J.A.V. (2016) - Obtenção de nanopartículas de peptídeos com atividade antioxidante de Spirulina sp. In: LEB. XXI de Engenharia Química, Fortaleza - CE. 25 a 29 de setembro 2016.

Lin, B.F.; Du, Y.M.; Liang, X.Q.; Wang, X.Y.; Wang, X.H. \& Yang, J.H. (2011) - Effect of chitosan coating on respiratory behavior and quality of stored litchi under ambient temperature. Journal of Food Engineering, vol. 102, n. 1, p. 94-99. https://doi.org/10.1016/j.jfoodeng.2010.08.009

Michalak, I.; Chojnacka, K. \& Saeid, A. (2017) - Plant Growth Biostimulants, Dietary Feed Supplements and Cosmetics Formulated with Supercritical CO2 Algal Extracts. Molecules, vol. 22, n. 1, art. 66. https://doi. org/10.3390/molecules22010066

Morais, M.G.; Stillings, C.; Dersch, R.; Rudisile, M.; Pranke, P.; Costa, J.A.V. \& Wendorff, J. (2015) Biofunctionalized Nanofibers Using Arthrospira (Spirulina) Biomass and Biopolymer. BioMed Research International, vol. 2015, art. 967814. https://dx.doi.org/10.1155/2015/967814

Nimisha, S.; Kherwar, D.; Ajay, K.M.; Singh, B. \& Usha, K. (2013) - Molecular breeding to improve guava (Psidium guajava L.): Current status and future prospective. Scientia Horticulturae, vol. 164, p. 578-588, https://doi.org/10.1016/j.scienta.2013.10.017

Oliveira, Á.M.F.; Rocha, R.H.C.; Guedes, W.A.; Dias, G.A. \& Lima, J.F. (2018) - Use of Chlorella sp. for coating 'tommy atkins' mango fruits stored under refrigeration. Semina: Ciências Agrárias, vol. 39, n. 2, p. 565-572. http://dx.doi.org/10.5433/1679-0359.2018v39n2p565

Onias, E.A; Rocha, R.H.C.; Lima, J.F. de; Onias, E.A. \& Furtunato, T.C.S. de (2016) - Organic 'Tommy Atkins' mango postharvest quality when treated with biofilms enriched by Spirulina platensis. Revista Cientifica, vol. 44, n. 3, p. 286-293. https://dx.doi.org/10.15361/1984-5529.2016v44n3p286-293 
Patil, V.; Chauhan, A.K. \& Singh, R.P. (2014) - Optimization of the spray-drying process for developing guava powder using response surface methodology. Powder Technology, vol. 253, p. 230-236. https://doi. org/10.1016/j.powtec.2013.11.033

Pentón-Rol, G.; Marín-Prida, J. \& Falcón-Cama, V.C. (2018) - Phycocyanin and Phycocyanobilin as Remyelination Therapies for Enhancing Recovery in Multiple Sclerosis and Ischemic Stroke: A Preclinical Perspective. Behavioral Sciences, vol. 8, n. 15, p. 1-17. https://doi.org/10.3390/bs8010015

Pereira, M.E.C.; Silva, A.S.; Santos, V.J.; Souza, E.G.; Ledo, C.A.S.; Lima, M.A.C. \& Amorim, T.B.F. (2009) - Aplicação de revestimento comestível para conservação pós-colheita da manga 'Tommy Atkins' em temperatura ambiente. In: Congresso Brasileiro Dd Fruticultura, 18, Florianópolis-SC.

Pinheiro, N.M.S. (2012) - Revestimento com cera de carnaúba incorporados de antimicrobianos em caju (Anacardium occidentale L) e goiaba (Psidium guajava). Tese de Doutorado, Viçosa, Universidade Federal de Viçosa. 122 p.

Queiroga, T.B. de (2016) - Qualidade pós-colheita de goiaba sob aplicação de biofilme à base de Chlorella sp. Monografia. Pombal, Universidade Federal de Campina Grande. 53 p.

Rana, S.; Siddiqui, S. \& Goyal, A. (2015) - Extension of the shelf life of guava by individual packaging with cling and shrink films. Journal of Food Science and Technology, vol. 52, n. 12, p. 8148-8155. https://doi. org/10.1007/s13197-015-1881-5

Salehi, M.; Moieni, A. \& Safaie, N. (2017) - A Novel Medium for Enhancing Callus Growth of Hazel (Corylus avellana L.). Scientific Reports, vol. 7, n. 1, p. 1 9. https://doi.org/10.1038/s41598-017-15703-z

Saboya, J.P.S.; Rodrigues, J.A.G. \& Farias, W.R.L. (2017) - Extração de ficocianinas de Spirulina platensis: opções metodológicas. Acta of Fisheries and Aquatic Resources, vol. 5, n. 2, p. 62-69. http://dx.doi.org/10.2312/ ActaFish.2017.5.2.62-69

Santana, D.B. (2015) - Respiração mitocondrial e mudanças físicas e químicas na pós-colheita de goiabas 'Paluma' e 'Cortibel'. Dissertação de Mestrado. Rio de Janeiro, Universidade Estadual do Norte Fluminense Darcy Ribeiro. 99 p.

Siqueira, A.P.O. (2012) - Uso de coberturas comestíveis na conservação pós-colheita de goiaba e maracujá-azedo. Dissertação de Mestrado. Rio de Janeiro, Universidade Estadual do Norte Fluminense Darcy Ribeiro.91 p.

Terada, M.; Watanabe, Y.; Kumitoma, M. \& Hayashi, E. (1978) - Differential rapid analyses of ascorbic acid and ascorbic acid 2-sulfate by dinitrophenylhydrazine method. Analytical Biochemistry, vol. 84, n. 2, p. 604-608. https://doi.org/10.1016/0003-2697(78)90083-0

Teuling, E.; Wierenga, P.A.; Schrama, J.W. \& Gruppen, H. (2017) - Comparison of Protein Extracts from Various Unicellular Green Sources. Journal of Agricultural and Food Chemistry, vol. 65, n. 36, p. 7989-8002. https://doi.org/10.1021/acs.jafc.7b01788

Torres, S.; Navia, R.; Murdy, R.C.; Cooke, P.; Misra, M. \& Mohanty, M. (2015) - Green Composites from Residual Microalgae Biomass and Poly (butylene adipate-co-terephthalate): Processing and Plasticization. ACS Sustainable Chemistry \& Engineering, vol. 3, n. 4, p. 614-624. https://doi.org/10.1021/sc500753h

Trindade, D.C.G. da; Lima, M.A.C. de; Silva, A.L. da; Assis, J.S. de; Sá, N.M.S.; Costa, R.S. \& Santos, P.S. (2004) - Armazenamento refrigerado de goiaba 'Paluma' submetida a atmosfera modificada e aplicação pós-colheita de 1-mcp. 4 p. [cit. 2017.06.07] https://www.alice.cnptia.embrapa.br/alice/bitstream/doc/154444/1/OPB830.pdf

Yu, J.; Hu, Y.; Xue, M.; Dun, Y.; Li, S.; Peng, N.; Liang, Y. \& Zhao, S. (2016) - Purification and Identification of Antioxidant Peptides from Enzymatic Hydrolysate of Spirulina platensis. Journal of Microbiology and Biotechnology, vol. 26, n. 7, p. 1216-1223. https://doi.org/10.4014/jmb.1601.01033

Vishwasrao, C. \& Ananthanarayan, L. (2016) - Postharvest shelf-life extension of pink guavas (Psidium guajava L.) using HPMC-based edible surface coatings. Journal of Food Science and Technology, vol. 53, n. 4, p. 1966-1974. https://dx.doi.org/10.1007\%2Fs13197-015-2164-x

Xing, Y.; Xu, Q.; Yang, S.X.; Chen, C.; Tang, Y.; Sun, S.; Zhang, L.; Che, Z. \& Li, X. (2016) - Preservation Mechanism of Chitosan-Based Coating with Cinnamon Oil for Fruits Storage Based on Sensor Data. Sensors, vol. 16, n. 1, p. 1-23. https://doi.org/10.3390/s16071111

Zhu, X.; Wang, Q.M.; Cao, J.K. \& Jiang, W.B. (2008) - Effects of chitosan coating on postharvest quality of mango (Mangifera indica L.cv. Tainong) fruits. Journal of Food Processing and Preservation, vol. 32, n. 5, p. 770-784. https://doi.org/10.1111/j.1745-4549.2008.00213.x 